\title{
Galactic Cosmic Ray Neon Isotopic Abundances Measured on ACE
}

\author{
W.R. Binns ${ }^{1}$, M.E. Wiedenbeck ${ }^{2}$, E.R. Christian ${ }^{3}$, A.C. Cummings ${ }^{4}$, J.S. George ${ }^{4}$, \\ P.L. Hink ${ }^{1}$, J. Klarmann ${ }^{1}$, R.A. Leske ${ }^{4}$, M. Lijowski ${ }^{1}$, S.M. Niebur ${ }^{1}$, \\ R.A. Mewaldt ${ }^{4}$, E.C. Stone ${ }^{4}$, T.T. von Rosenvinge ${ }^{3}$, and N.E. Yanasak ${ }^{2}$ \\ 1. Washington University, St. Louis, MO 63130 USA \\ 2. Jet Propulsion Laboratory, Pasadena, CA 91109 USA \\ 3. NASA/Goddard Space Flight Center, Greenbelt, MD 20771 USA \\ 4. California Institute of Technology, Pasadena, CA 91125 USA
}

\begin{abstract}
Measurements of neon isotopic abundances from the ACE-CRIS experiment are presented. Abundances have been obtained in six energy intervals over the energy range of $\sim 100 \leq \mathrm{E} \leq 280 \mathrm{MeV} /$ nucleon. These measurements are compared with the ACE-SIS data for lower energies extending down to $\sim 8 \mathrm{MeV} /$ nucleon. We find that the CRIS ${ }^{22} \mathrm{Ne} /{ }^{20} \mathrm{Ne}$ abundance ratio at the source is a factor of $5.0 \pm 0.3$ greater than for the solar wind. The CRIS measured abundances agree well with previous experiments. The CRIS and SIS measurements are in good agreement for the higher SIS energies. However for the lower energy SIS data, where the anomalous cosmic rays (ACR) are being sampled, the ratio decreases and agrees with solar wind abundances. The implications of these results are discussed.
\end{abstract}

\section{INTRODUCTION}

The ${ }^{22} \mathrm{Ne} /{ }^{20} \mathrm{Ne}$ ratio in the galactic cosmic rays (GCR) has been shown by several experiments (1-4) to be overabundant when compared to solar wind (SW) abundances (5), which is the sample of solar system (SS) matter taken to best represent the composition of material from which the solar system formed (6). Cassé and Paul (7) suggested that this overabundance could be due to the presence of an admixture of WolfRayet (WR) star material mixed into the general GCR source material. Woosley and Weaver (8) developed a "supermetallicity" model that recognizes that the synthesis of neutron-rich isotopes in massive stars is directly proportional to their initial metallicity (fraction of elements heavier than He). If GCRs originate in a region of the Galaxy with higher metallicity than the SS, this could result in an overabundance of ${ }^{22} \mathrm{Ne} /{ }^{20} \mathrm{Ne}$. However, an overabundance of the neutron rich isotopes of $\mathrm{Mg}$ and Si should also be observed, and that is not the case. Olive and Schramm (9) suggest that instead of the GCR ${ }^{22} \mathrm{Ne} /{ }^{20} \mathrm{Ne}$ ratio being anomalously high, it is actually the solar system that is anomalously low. This could result if supernovae in the near vicinity at the time of formation of the solar system injected large amounts of ${ }^{20} \mathrm{Ne}$ into the pre-solar nebula. These models are reviewed by Mewaldt (10). Recently
Soutoul and Legrain (11) have suggested that in diffusion models GCRs may come preferentially from the inner Galaxy if the cosmic ray density is greater toward the center of the Galaxy. (Gamma-ray observations weakly support a GCR density gradient (12)). In this model the GCRs sample material preferentially from higher metallicity regions closer to the galactic center than the SS.

In this paper we present measurements of the $\mathrm{Ne}$ isotopes obtained by the Cosmic Ray Isotope Spectrometer (CRIS) instrument on the ACE spacecraft (13). We have obtained the ${ }^{22} \mathrm{Ne} /{ }^{20} \mathrm{Ne}$ ratio energy dependence for the energy range $100 \leq \mathrm{E} / \mathrm{M} \leq 281 \mathrm{MeV} /$ nucleon and compare these data with those from the ACE-Solar Isotope Spectrometer (SIS) instrument (14) for lower energies. At the lowest energies ( 8-35 MeV/nucleon) SIS measures the anomalous cosmic rays (ACR) that are believed to be a sample of the very-local interstellar-medium (VLISM). Thus we can compare the $\mathrm{Ne}$ isotopic composition in the GCR source, which is believed to be a sample of the ISM over a significant part of the galactic disk, with that of the VLISM. The CRIS abundance ratios are compared with results from other experiments. We also derive the ${ }^{22} \mathrm{Ne}^{20} \mathrm{Ne}$ source ratio using ${ }^{21} \mathrm{Ne}$ measured abundances as a "tracer" (15) for secondary production of the neon isotopes. 


\section{MEASUREMENTS}

In Figure 1a-f we show mass histograms of the CRIS neon data for events in 6 energy bins spanning the energy range of $100<\mathrm{E} / \mathrm{M}<281 \mathrm{MeV} /$ nucleon.

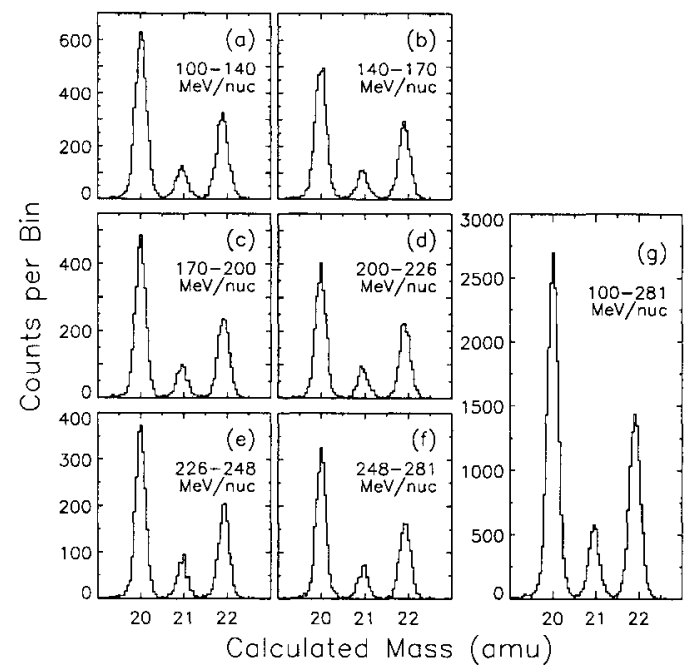

FIGURE 1. Mass histograms of neon events in 6 energy bins (a-f). Figure $1 \mathrm{~g}$ is the sum of all events in Figs. la-f.

Figure 1g shows combined data from the 6 energy intervals. These events are a selected, high-resolution data set that were collected from Jan. 21, 1998 through Sept. 24,1999 . The primary selection was to use only events with angle $\leq 25^{\circ}$ relative to the detector normal. This selected data set corresponds to $\sim 35 \%$ of the good events for all angles. The mass resolution is sufficiently good $(0.10 \mathrm{amu}$ for events plotted in Figure 1) so that there is only a very small overlap of the mass distributions for adjacent masses. To obtain abundances, we have taken mass cuts at the minimum between peaks and simply counted events. The number of counts in the full data set is $3.0 \times 10^{4}$. The statistical accuracy of the CRIS and SIS data are such that the neon isotopic abundances as a function of energy can be studied in greater detail than was previously possible.

In Figure 2 we plot the ${ }^{22} \mathrm{Ne} /{ }^{20} \mathrm{Ne}$ ratio measured by CRIS (circles) and by SIS (16) (squares) respectively. (The CRIS measured values are corrected so that the E/M range for each data point is equal for the neon isotopes). We also show the SW abundance ratio as a horizontal dotted line $(5,6)$. The CRIS GCR ${ }^{22} \mathrm{Ne} /{ }^{20} \mathrm{Ne}$ ratio is approximately constant with a small decrease in the ratio toward lower energies, and is considerably enhanced over the SW ratio. SIS measures a mix of GCRs (dominant at the higher SIS energies) that have a high ${ }^{22} \mathrm{Ne} /{ }^{20} \mathrm{Ne}$ ratio, and ACRs (dominant at the lower SIS energies) that have a ratio similar to SW $(16,17)$. Thus we see that there is a dramatic difference between the CRIS GCR and the low-energy SIS ACR measurements.

The ${ }^{21} \mathrm{Ne} /{ }^{20} \mathrm{Ne}$ ratios measured by CRIS are plotted in Figure 3. The data show a roughly constant ratio with a slight decline at lower energies as was the case for ${ }^{22} \mathrm{Ne} /{ }^{20} \mathrm{Ne}$. The $\mathrm{SW}{ }^{21} \mathrm{Ne} /{ }^{20} \mathrm{Ne}$ ratio is very low $\left(2.4 \times 10^{-3}(5,6)\right)$ and the much higher abundance of ${ }^{21} \mathrm{Ne}$ in GCRs is thus believed to be almost entirely secondary (i.e. resulting from nuclear interactions of primary cosmic rays during propagation). This makes it possible to use ${ }^{21} \mathrm{Ne}$ as a nearby "tracer" to estimate the fractions of ${ }^{22} \mathrm{Ne}$ and ${ }^{20} \mathrm{Ne}$ that are secondary GCR nuclei (15).

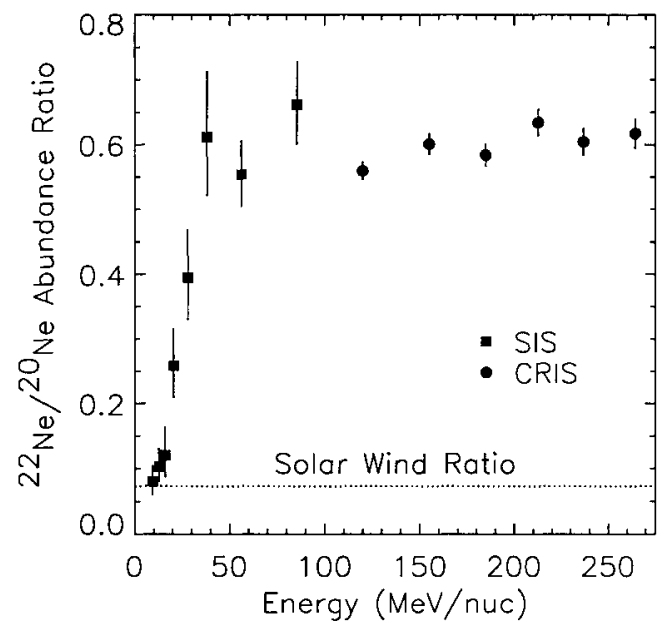

FIGURE 2. The ${ }^{22} \mathrm{Ne} /{ }^{20} \mathrm{Ne}$ ratio is plotted as a function of energy for CRIS (circles) and SIS (16) (squares).

In Figure 4 we plot the CRIS Ne isotopic ratios averaged over the full energy range (taken from Fig $1 \mathrm{~g}$ ) and compare them with measurements made by other experiments (1-4). We have adjusted the ratios of the other experiments to correspond to the CRIS modulation level $(\sim 500 \mathrm{MV})$. The CRIS measurements have smaller statistical uncertainties than previous experiments (the CRIS error bars are smaller than the data points), and there is reasonably good agreement with those measurements.

We have corrected for secondary production during propagation using the tracer method (15) to obtain the source abundance ratio for ${ }^{22} \mathrm{Ne} /{ }^{20} \mathrm{Ne}$. In the tracer method the observed abundances of secondary nuclides such as ${ }^{21} \mathrm{Ne},{ }^{19} \mathrm{~F}$, or ${ }^{17} \mathrm{O}$ are scaled to determine the secondary contribution to the observed ${ }^{22} \mathrm{Ne}$, and thereby to derive the ${ }^{22} \mathrm{Ne}$ source abundance. 


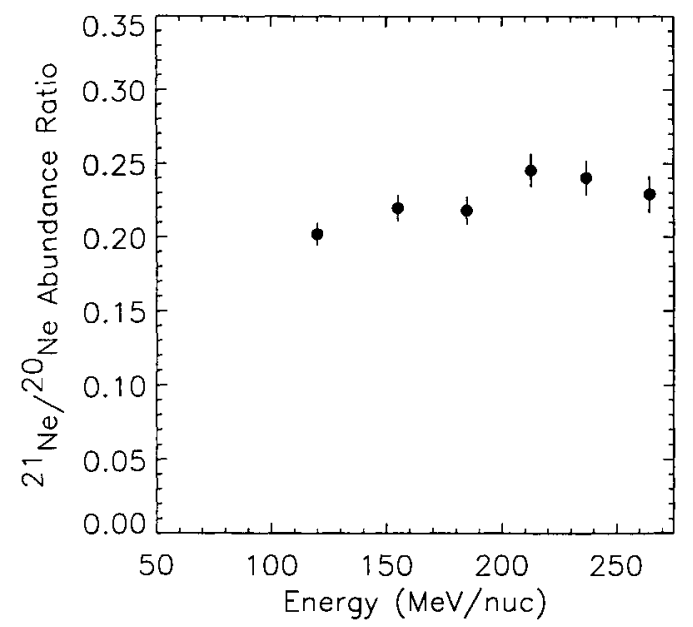

FIGURE 3. The ${ }^{21} \mathrm{Ne} /{ }^{20} \mathrm{Ne}$ ratio measured by CRIS is plotted as a function of energy.

A series of leaky-box propagation calculations was carried out using various assumed escape mean free paths. Figure 5 shows the calculated relationship between the ${ }^{22} \mathrm{Ne} /{ }^{20} \mathrm{Ne}$ source ratio and the abundance of the secondary nuclide ${ }^{21} \mathrm{Ne}$ at Earth predicted by the model (plotted as a difference from the observed value). The point on the abscissa corresponding to a $0 \%$ difference for ${ }^{21} \mathrm{Ne}$ at Earth gives the best estimate of the ${ }^{22} \mathrm{Ne}$ source abundance using that secondary as a constraint. Source abundances obtained using ${ }^{19} \mathrm{~F}$ and ${ }^{17} \mathrm{O}$ secondary tracers were also obtained (not shown) and averaged with the ${ }^{21} \mathrm{Ne}$ value to obtain a best overall ratio. The measurement uncertainty in the ${ }^{21} \mathrm{Ne}$ abundance is represented by the vertical dotted lines in Figure 5. The points at which they intersect the plotted line indicate the uncertainty in the ${ }^{22} \mathrm{Ne} /{ }^{20} \mathrm{Ne}$ ratio due to the uncertainty in the ${ }^{21} \mathrm{Ne}$ measurement.

In Figure 6 we plot the preliminary CRIS source abundance of ${ }^{22} \mathrm{Ne} /{ }^{20} \mathrm{Ne}$ that is $0.366 \pm 0.006 \pm 0.014$. The source abundance ratio was calculated by averaging the source ratio estimates obtained from the three tracer isotopes $\left({ }^{19} \mathrm{~F}\right.$ and ${ }^{17} \mathrm{O}$ tracers both give source abundance ratios of 0.375 compared to 0.350 for the ${ }^{21} \mathrm{Ne}$ tracer source estimate in Figure 5). The first uncertainty in the abundance ratio is the quadratic sum of the ${ }^{22} \mathrm{Ne} /{ }^{20} \mathrm{Ne}$ ratio uncertainty from counting statistics and the uncertainty from counting statistics for ${ }^{21} \mathrm{Ne}$ (Figure 5). The second uncertainty is our estimate of the systematic uncertainty calculated using the "sample standard deviation" obtained from the three tracer isotope estimates. We note that the differing source ratio estimates from the three different tracer isotopes and the derived systematic uncertainty are comparable to that expected from nuclear crosssection uncertainties. The error bar on the CRIS data

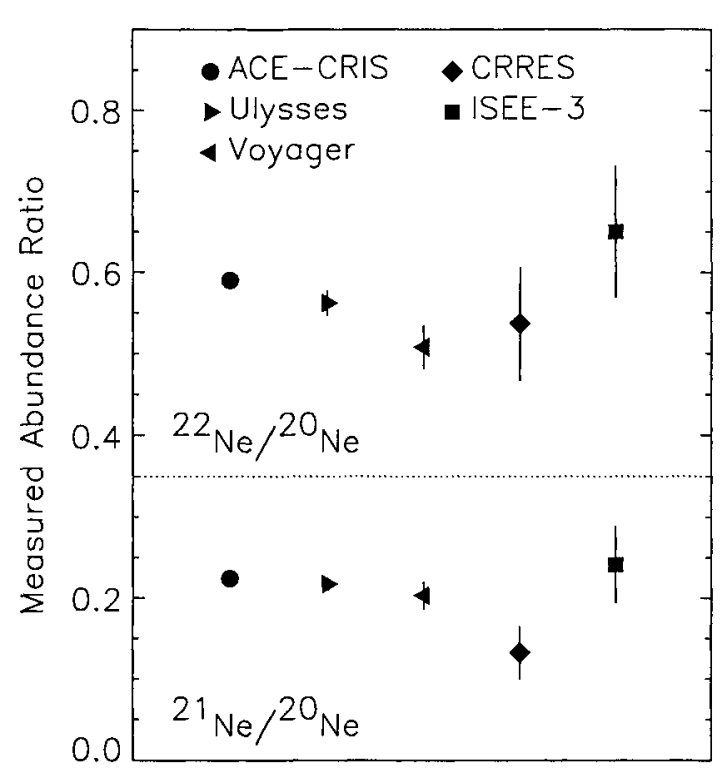

FIGURE 4. Ratios of ${ }^{22} \mathrm{Ne} /{ }^{20} \mathrm{Ne}$ and ${ }^{21} \mathrm{Ne} /{ }^{20} \mathrm{Ne}$ measured by ACE-CRIS (circle), Ulysses (2)(right triangle), Voyager (3)(left triangle), CRRES (4)(diamond), and ISEE-3 (1)( square). Ratios have been adjusted for solar modulation (4).

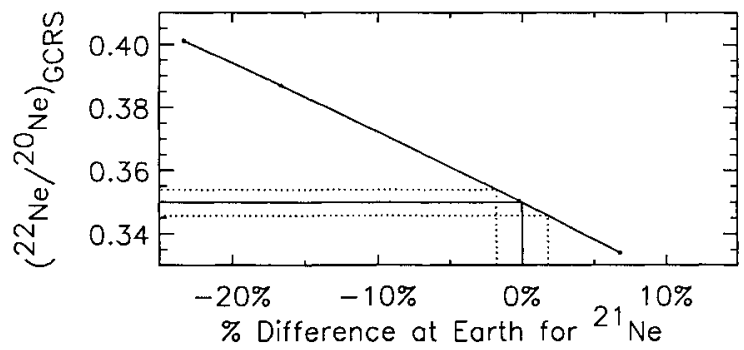

FIGURE 5. The plotted curve shows the value of the ${ }^{22} \mathrm{Ne} /{ }^{20} \mathrm{Ne}$ source ratio as a function of percent difference between the calculated value of the tracer isotope ${ }^{21} \mathrm{Ne}$ and our measured value. The horizontal dotted lines indicate the uncertainty in the ${ }^{22} \mathrm{Ne} /{ }^{20} \mathrm{Ne}$ source estimate due to our measurement uncertainty for ${ }^{21} \mathrm{Ne}$ (vertical dotted lines).

point in Figure 6 is the linear sum of the statistical and systematic uncertainties.

\section{DISCUSSION}

The values for the SW (5), which are believed to give the best estimate of the solar system neon abundances (6), a range of values for SEPs (18), and meteoritic abundances (19) are plotted in Figure 6. The GCR ${ }^{22} \mathrm{Ne} /{ }^{20} \mathrm{Ne}$ ratio is $5.0 \pm 0.3$ times the $\mathrm{SW}$ value of 0.073 (5). Leske et al. (17) give similar results obtained by SAMPEX. Neon, unlike most other elements, is observed to have a number of separate 
populations in the solar system with distinctly different isotopic compositions. Ne-A is found in carbonaceous chondrite meteorites and may be pre-solar in origin. $\mathrm{Ne}-\mathrm{B}$ is believed to result from solar wind implantation in grains and has a ${ }^{22} \mathrm{Ne} /{ }^{20} \mathrm{Ne}$ ratio very close to that of contemporary measurements of the solar wind (5). Ne-E, which is nearly pure ${ }^{22} \mathrm{Ne}(19)$ is found in $\mathrm{SiC}$ and Graphite grains and is believed to come from He burning $\mathrm{AGB}$ stars and the decay of ${ }^{22} \mathrm{Na}$ produced in $\mathrm{SN}$ or novae. It is the only component found in meteorites that has a ${ }^{22} \mathrm{Ne} /{ }^{20} \mathrm{Ne}$ ratio greater than that of the GCRs.

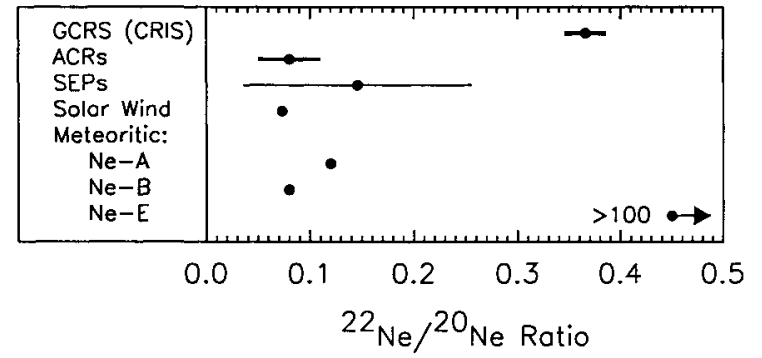

FIGURE 6. ${ }^{22} \mathrm{Ne} /{ }^{20} \mathrm{Ne}$ source abundance for CRIS (GCR) and SIS (ACR) data compared to SW $(5,6)$, SEPs (18) and meteoritic abundances (19). The error bar plotted for CRIS is the linear sum of the statistical and systematic uncertainties.

The fact that the GCRs show a marked enhancement in ${ }^{22} \mathrm{Ne} /{ }^{20} \mathrm{Ne}$ while the ACRs show good agreement with solar wind abundances is remarkable and is consistent with the SAMPEX results (17). If the ACR ratio is representative of the general ISM, this would indicate that there is a large overabundance of high-energy ${ }^{22} \mathrm{Ne}$ relative to ${ }^{20} \mathrm{Ne}$ in the Galaxy (i.e. cosmic rays) compared to low energy matter (i.e. dust grains and gas). We note that it is not known at present to what extent the ACRs actually represent the general ISM. It is also important to note that the Solar System may not have formed in the local ISM. Based on the higher metallicity of the sun relative to that of nearby stars, Wielen et al (20) suggest that the sun formed $\sim 2 \mathrm{kpc}$ closer to the Galactic center than our present position at Galactocentric radius of $\sim 8.5 \mathrm{kpc}$.

This overabundance in the GCRs could result from the acceleration of WR star material (7) since the high velocity winds which result in mass losses of $\sim 10^{-5}$ solar masses per year expel large quantities of Heburning material rich in ${ }^{22} \mathrm{Ne}(21,22)$. Since these massive stars have short lifetimes on average before they become supernovae $\left(\sim 10^{5}\right.$ y) (23) some of this material may be swept up and accelerated by nearby $\mathrm{SN}$, or by the supernova shock from the star that ejected the material in the first place. This would occur without substantial mixing into the ambient ISM since $\sim 90 \pm 10 \%$ of WR stars are believed to exist in $\mathrm{OB}$ associations within superbubbles formed in giant molecular clouds (21) (based upon observations by van Dyk et al (24) on core collapse supernovae and comments by Higdon et al. (23)). Thus it would seem that WR stars in superbubbles might be an ideal astrophysical setting for WR material to provide the enhanced ${ }^{22} \mathrm{Ne} /{ }^{20} \mathrm{Ne}$ observed in GCRs.

\section{ACKNOWLEDGMENTS}

This research was supported by the National Aeronautics and Space Administration at Washington University, the California Institute of Technology (under grant NAG5-6912), the Jet Propulsion Laboratory, and the Goddard Space Flight Center. It was also supported by the McDonnell Center for the Space Sciences at Washington University.

\section{REFERENCES}

1. Wiedenbeck, M.E., and Greiner, D.E., Phys. Rev. Lett., 46, 682, 1981.

2. Connell, J.J., and Simpson, J.A., Proc. of the 25th International Cosmic Ray Conference, 3, 381, 1997.

3. Lukasiak, A. et al, ApJ, 426, 366, 1994.

4. DuVernois, M.A. et al, $A p J, 466,457,1996$.

5. Geiss, J., Proc. of the 13th International Cosmic Ray Conference, 5, 3375, 1973.

6. Anders, E, and Grevesse, N., Geochim. Et Cosmochim. Acta 53, 197, 1989.

7. Cassé, M., and Paul, J.A., ApJ, 258, 860, 1982.

8. Woosley, S.E., and Weaver, T.A., Ap.J., 243, 651,1981.

9. Olive, K.A., and Schramm, D.N., Ap.J., 257, 276, 1982.

10. Mewaldt, R.A., in Cosmic Abundances of Matter, Ed. By C.J. Waddington, AIP Conference Proc. 183, 124, 1988.

11. Soutoul, A., and Legrain, R. Proc. of the 26th International Cosmic Ray Conference, 4, 180, 1999.

12. Hunter, S.D., et al, ApJ 481, 205 (1997).

13. Stone, E.C., et al, Space Science Reviews, 86, 285, 1998.

14. Stone, E.C. et al, Space Science Reviews 86, 357, 1998.

15. Stone, E.C., and Wiedenbeck, M.E., ApJ 231, 606, 1979.

16. Leske, R.A. et al, Proc. of the 26th International Cosmic Ray Conference, 7, 539, 1999.

17. Leske, R.A. et al, Space Sci. Revs. 78, 149, 1996.

18. Leske, R.A. et al, GRL, 26, 2693, 1999.

19. Ozima, M., and Podosek, F.A., Noble Gas Geochemistry, Cambridge: Cambridge University Press, 1983.

20. Wielen, R., Fuchs, B., and Dettbarn, C, A, Astron. Astrophys., 314, 438, 1996.

21. Maeder, A. and Meynet, G., Astron. Astrophys., 278, 406, 1993.

22. Chiosi, C, and Maeder, A, Ann. Rev. Astron. Astrophys, 24, 329, 1986.

23. Higdon, J.C., Lingenfelter, R.E., and Ramaty, R., ApJ, 509, L33, (1998), and Private Communication.

24. Van Dyk, S.D. et al, $A J, 111,2017,1996$. 